\title{
High Energy Resolution Off-Resonant Spectroscopy for X-Ray Absorption Spectra Free of Self-Absorption Effects
}

\author{
W. Błachucki, ${ }^{1,}{ }^{*}$ J. Szlachetko, ${ }^{2,3}$ J. Hoszowska, ${ }^{1}$ J.-Cl. Dousse, ${ }^{1}$ Y. Kayser ${ }^{1, \dagger}$ M. Nachtegaal, ${ }^{2}$ and J. Sá ${ }^{2}$ \\ ${ }^{1}$ Department of Physics, University of Fribourg, CH-1700 Fribourg, Switzerland \\ ${ }^{2}$ Paul Scherrer Institute, CH-5232 Villigen-PSI, Switzerland \\ ${ }^{3}$ Institute of Physics, Jan Kochanowski University, 25-406 Kielce, Poland
}

(Received 13 December 2013; published 30 April 2014)

\begin{abstract}
$\mathrm{X}$-ray emission spectra recorded in the off-resonant regime carry information on the density of unoccupied states. It is known that by employing the Kramers-Heisenberg formalism, the high energy resolution off-resonant spectroscopy (HEROS) is equivalent to the x-ray absorption spectroscopy (XAS) technique and provides the same electronic state information. Moreover, in the present Letter we demonstrate that the shape of HEROS spectra is not modified by self-absorption effects. Therefore, in contrast to the fluorescence-based XAS techniques, the recorded shape of the spectra is independent of the sample concentration or thickness. The HEROS may thus be used as an experimental technique when precise information about specific absorption features and their strengths is crucial for chemical speciation or theoretical evaluation.
\end{abstract}

DOI: 10.1103/PhysRevLett.112.173003

PACS numbers: 32.30.Rj, 31.15.ag, 32.70.Jz, 32.80.Fb

The high-intensity and highly monochromatic x-ray radiation available at synchrotron sources allow researchers from different disciplines to study the local electronic and geometric structure of materials by means of x-ray absorption spectroscopy (XAS). To record a XAS spectrum the $\mathrm{X}$-ray energy is tuned around the electron binding energy of the element of interest, thereby exciting electrons from one of the core levels to unoccupied states or into the continuum. The photo-absorption process depends on the photon energy and is a measure of the material's absorption coefficient which in turn provides information on the material properties. The energy region around the absorption edge, namely the $\mathrm{x}$-ray absorption near edge structure (XANES), reflects the density of empty states [1]. The region starting about $100 \mathrm{eV}$ above the absorption edge is called the extended $\mathrm{x}$-ray absorption fine structure (EXAFS) region, and the analysis of this region yields information on the local geometric structure [2].

$\mathrm{X}$-ray absorption spectra can be measured in the transmission, fluorescence, and electron-yield mode [3,4]. In the transmission mode, the XAS spectra are measured by recording the intensity of the photon beam before and after the sample. The fluorescence mode involves the use of $\mathrm{x}$-ray emission spectroscopy (XES) techniques allowing detection of the fluorescence yield as a function of the incident photon energy. The XAS spectrum can be obtained from either the total fluorescence yield (TFY) or the partial fluorescence yield (PFY) measurements. In TFY the fluorescence intensity is integrated over a broad emission energy range, while in the PFY it is integrated over a selected emission energy range. In particular, the fluorescence yield integrated over an energy band centered at a given fluorescence line and narrower than the natural linewidth of the latter is referred to as the high energy resolution XAS [5,6]. It provides more detailed information about absorption features and allows precise chemical speciation [7].

The field of $\mathrm{x}$-ray absorption spectroscopy is a very important tool in the fields of physics, chemistry, medicine, biology, and material sciences (see, e.g., Refs. [8-14]). In view of the wide range of applications of XAS-based techniques in different disciplines, considerable progress on the experimental side and a large amount of effort to account for self-absorption effects in XAS spectra measurements has been made. It has been long established that conventional fluorescence mode XAS measurements suffer from the self-absorption process in the target material [15]. The self-absorption occurs when the induced fluorescence radiation is partially absorbed in the sample on its way out. The measured fluorescence yield as a function of the photon energy depends thus not only on the sample properties but also on the photon penetration depth and the escape length.

Different approaches to obtain fluorescence data unaffected by this phenomenon have been proposed so far. Ablett et al. [16] as well as Booth and Bridges [17], independently, reported on an analytic method to correct the measured XAS spectra for self-absorption effects. Their approach, however, assumes that the linear attenuation coefficient of the material under study is known, whereas measurements of linear absorption coefficients require in principle XAS spectra already free of self-absorption effects. Recently, Achkar et al. [18,19] introduced the inverse partial fluorescence yield (IPFY) method which relies on measuring the $\mathrm{x}$-ray emission fluorescence from an element or core-hole excitation that is different from the 
absorption edge being probed with the incident beam. The self-absorption effects are avoided because the principle behind IPFY resides in using nonresonant $\mathrm{X}$-ray emission to obtain an XAS spectrum. They demonstrated that when irradiating a single crystal of $\mathrm{La}_{1.475} \mathrm{Nd}_{0.4} \mathrm{Sr}_{0.125} \mathrm{CuO}_{4}$ with photon energies around the $\mathrm{Cu} L_{2,3}$ and $\mathrm{Nd} M_{4,5}$ absorption edges, the inverse of the $\mathrm{O} K \alpha \mathrm{PFY}$ spectrum provides XAS spectra of $\mathrm{Cu}$ and $\mathrm{Nd}$ around their $L_{2,3}$ and $M_{4,5}$ absorption edges, respectively, free of self-absorption effects [18]. Nonetheless, in many scientific cases the self-absorption problem cannot be circumvented because of the nature of the sample itself whose mass thickness may not be easily reduced either by thinning or diluting. In conclusion, one can state that mitigation of self-absorption has been and still is a topic of active research (see, e.g., Refs. [18-21]).

As recently demonstrated, an alternative approach to measure XAS spectra in the fluorescence mode is the high energy resolution off-resonant spectroscopy (HEROS) [22-24]. This technique is based on theoretical investigations of Tulkki and Åberg [25]. It combines the irradiation of the sample at a fixed incident beam energy, detuned to below an absorption edge, with the detection of the x-ray fluorescence by means of a dispersive-type spectrometer. Thanks to that, the unoccupied electronic states may be probed using a fixed optical arrangement. The very attractive feature of the off-resonant technique is that the incoming beam energy is fixed and of a single energy. In addition, the energies of both the incident beam and the fluorescence radiation are below the investigated absorption threshold. In this Letter, we present the $L_{3}$ absorption edge spectrum of elemental Ta measured by means of different XAS techniques. Ta was chosen because the XAS $L_{3}$ white line which is very sensitive to the self-absorption effect is particularly strong for this element. We demonstrate that the spectral features in the XAS spectra measured in the transmission and the fluorescence mode change with the sample thickness, while the HEROS spectra show no dependence. The change of the sample thickness (concentration) results solely in the entire HEROS yield modulation.

The measurements were carried out at the SuperXAS beam line of the Swiss Light Source of the Paul Scherrer Institute, Switzerland. The collimated x-ray beam was monochromatized by means of a double $\operatorname{Si}(111)$ crystal monochromator and focused by a toroidally bent $\mathrm{Rh}$ mirror on the sample to a spot size of $100 \times 100 \mu \mathrm{m}^{2}$. The photon flux was $7-8 \times 10^{11}$ photons/second with a relative energy resolution of $1.4 \times 10^{-4}$. Nine Ta metallic foils of nominal thicknesses in the range $0.5-50 \mu \mathrm{m}$ were irradiated at ambient conditions at an incident angle of $45^{\circ}$. The Ta $L \alpha_{1,2}$ XES spectrum was detected by means of a wavelength-dispersive spectrometer [26] based on the von Hamos geometry consisting of a cylindrically curved segmented-type $\mathrm{Si}(444)$ crystal and the position-sensitive microstrip silicon MYTHEN detector [27]. From a Gaussian fit to the elastic scattering peak, the full width at half maximum (FWHM) experimental resolution of $1.3 \mathrm{eV}$ was found. For transmission mode measurements two 30-cm-long, helium filled, ionization chambers were used. For each sample, the $L_{3}$ absorption edge XAS spectrum was measured in the transmission and fluorescence mode simultaneously. The TFY and high-resolution XAS spectra were obtained from the $L \alpha_{1,2}$ XES data measured for x-ray beam energies tuned around the $L_{3}$ edge. The off-resonant emission spectrum was collected at a beam energy of $26 \mathrm{eV}$ below the $L_{3}$ edge. The acquisition time for each XES spectrum was $10 \mathrm{~s}$ and for the HEROS spectrum $6000 \mathrm{~s}$.

The principle of HEROS is based on the KramersHeisenberg formula modified by Tulkki and Åberg $[25,28]$, which describes the differential cross section of the resonant inelastic X-ray scattering (RIXS) process [29]. Schematic energy diagrams illustrating RIXS are presented in Fig. 1. For photon energies above the absorption edge threshold [see Fig. 1(a)], the excitation of a core-level electron into an unoccupied state above the Fermi level is followed by the deexcitation of another core-level electron and the emission of $\mathrm{x}$-ray fluorescence. The resulting fluorescence spectrum is broadened by both the initial and the final state widths. In the off-resonant regime, as the energy of the incident photon is not high enough to promote the inner-shell electron above the Fermi level, the emitted photon gives away part of its energy to the photoelectron in the scattering process. The energy of the emitted photon is thus reduced with respect to the fluorescence line energy, and the resulting XES spectrum carries information on the density of unoccupied states, as illustrated in Fig. 1(b). Because of the coherence of the core-hole excitation and deexcitation in the off-resonant
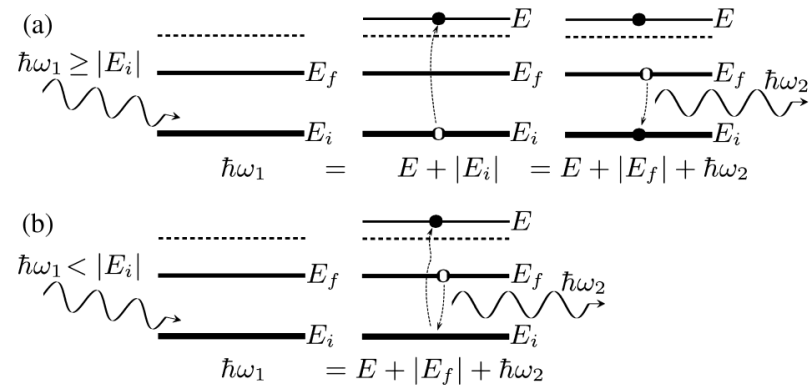

FIG. 1. Schematic of the RIXS process for an incident photon energy $\hbar \omega_{1}$ involving excitation of an electron from the initial state of energy $E_{i}$ to an unoccupied state $E$ above the Fermi level, and electron deexcitation from the final state of energy $E_{f}$ accompanied by the emission of a photon with the energy $\hbar \omega_{2}$. For incident photon energies greater or equal to the absorption edge energy, the $\mathrm{x}$-ray scattering can be considered as a two-step process and $\hbar \omega_{2}=\left|E_{i}\right|-\left|E_{f}\right|$ (a). Otherwise, the excitation and deexcitation occur coherently and a one-step picture applies, where $\hbar \omega_{2}(E)=\hbar \omega_{1}-\left|E_{f}\right|-E<\left|E_{i}\right|-\left|E_{f}\right|$ (b). 
conditions, the initial state width does not contribute to the XES spectrum broadening.

Assuming that the intensity of the emitted radiation is proportional to the scattering cross section and the oscillator strength distribution for electron excitation to the density of unoccupied states, for off-resonant excitation well below the ionization threshold one can reformulate the equation of Tulkki and Åberg [25] as follows [22]:

$$
\begin{aligned}
I_{\mathrm{XES}}\left(\hbar \omega_{2}\right) \sim & \int_{0}^{\infty}\left[\frac{\hbar \omega_{2}}{\hbar \omega_{1}} \frac{\left(\left|E_{i}\right|-\left|E_{f}\right|\right)\left(E+\left|E_{i}\right|\right)}{\left(E+\left|E_{i}\right|-\hbar \omega_{1}\right)^{2}+\Gamma_{i}^{2} / 4}\right. \\
& \left.\times I_{\mathrm{XAS}}(E) \delta\left(\hbar \omega_{1}-\left|E_{f}\right|-E-\hbar \omega_{2}\right)\right] d E,
\end{aligned}
$$

where $I_{\mathrm{XES}}\left(\hbar \omega_{2}\right)$ denotes the emission spectrum, $I_{\mathrm{XAS}}(E)$ stands for the XAS spectrum as a function of the excited photoelectron energy $E$, and $\Gamma_{i}$ is the initial state broadening; the meaning of the remaining symbols is the same as in Fig. 1. The final state broadening is omitted. Eq. (1) thus becomes readily transformable and allows retrieving from the off-resonant XES data $I_{\mathrm{XES}}\left(\hbar \omega_{2}\right)$ the XAS spectrum which is proportional to the linear absorption coefficient $\mu$ as a function of the photon energy $\left(E+\left|E_{i}\right|\right)$. Note that $\mu\left(E+\left|E_{i}\right|\right)=\mu\left(\hbar \omega_{1}-\hbar \omega_{2}+\left|E_{i}\right|-\left|E_{f}\right|\right)$ according to the energy conservation relation (see Fig. 1) $[25,30]$.

In Fig. 2 we present XAS spectra of elemental tantalum around its $L_{3}$ absorption edge for different sample thicknesses recorded in the transmission mode, TFY, and high energy resolution. In the transmission mode spectra, plotted in Fig. 2(a), the white line is observable only for samples up to a thickness of $20 \mu \mathrm{m}$. It was found that the diminution of the white line intensity is accompanied by an $2 \mathrm{eV}$ increase of its FWHM. For thicker samples also a decrease of the overall spectral intensity resulting in an apparent shift of the absorption edge energy with the sample thickness is clearly seen. This well-known effect is named the "overabsorption" or "thickness" effect [31] or the "incident beam self-absorption" [32]. It arises from too high values of the product of the linear attenuation coefficient and the sample thickness. As a result, the transmitted photon intensity is no longer proportional to the photoabsorption cross section as the photon energy is varied across the edge. In order to avoid the overabsorption Stern and Kim [33] recommend to use sample thicknesses $d$ satisfying the condition $\mu_{\max } d \leq 1$, where $\mu_{\max }$ stands for the maximum value of the absorption coefficient. Indeed, from among the studied Ta samples only the $0.5 \mu \mathrm{m}$ and $1 \mu \mathrm{m}$ thick ones comply with this recommendation while for the thickest one the $\mu_{\max } d$ product reaches a value of about 42 .

The Ta $L_{3}$ edge high energy resolution and TFY XAS spectra are depicted in Fig. 2(b) and Fig. 2(c), respectively, and in Fig. 2(d) a RIXS map is shown for illustration. Self-absorption effects are predominantly reflected in the
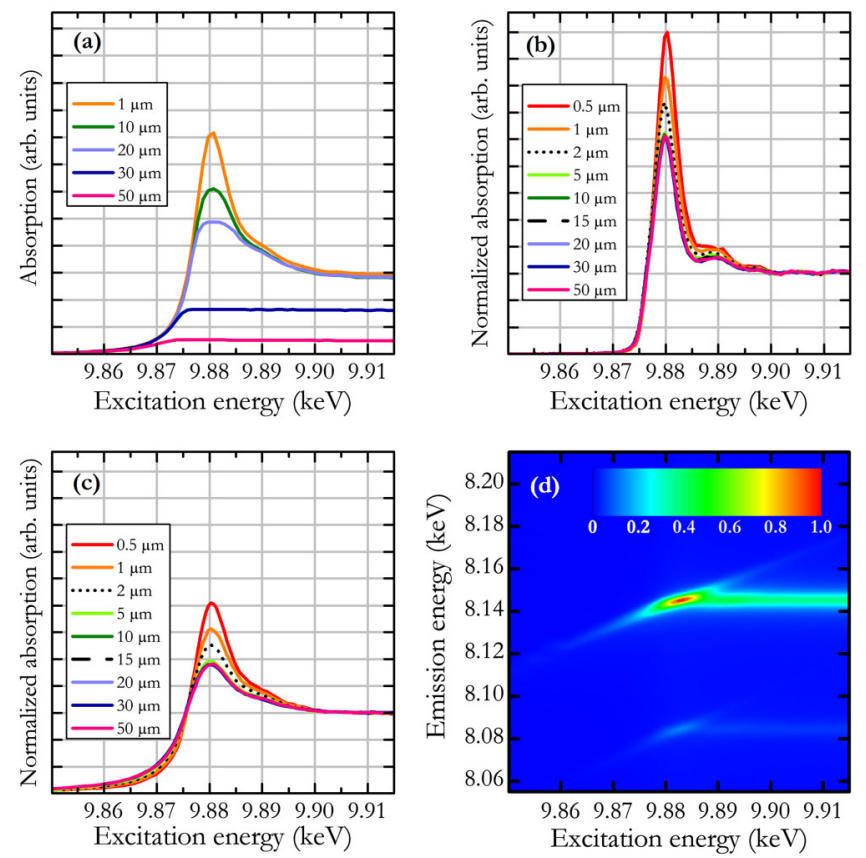

FIG. 2 (color online). Ta $L_{3}$ absorption edge (9.881 keV) scanned with an $\mathrm{x}$-ray beam of energy varying in the range 9.850-9.915 keV. The data were recorded for samples of different thicknesses both in transmission (a) and fluorescence mode in high energy resolution (b) and TFY (c). The spectra depicted in (b) and (c) were normalized to coincide at $9.905 \mathrm{keV}$. High energy resolution XAS spectra were obtained by integrating the XES data within a $2 \mathrm{eV}$ energy window centered at the Ta $L \alpha_{1}\left(L_{3} M_{5}\right)$ fluorescence line $(8.146 \mathrm{keV})$, while TFY XAS spectra result from integrating the XES data within the whole Ta $L \alpha_{1,2}\left(L_{3} M_{5,4}\right)$ domain. (d) RIXS plane recorded for the $10 \mu \mathrm{m}$ thick sample showing the integration area (dotted lines) for the high energy resolution XAS. Note that the integration energy interval is smaller than the initial state lifetime broadening of $4.68 \mathrm{eV}$.

intensity reduction of the white line and the broadening of the latter and to a smaller extent in distortions of the XANES peaks. Differences in the absorption spectra are only observable for sample thicknesses up to $10 \mu \mathrm{m}$. The recorded off-resonant XES and the reconstructed HEROS XAS spectra are shown in Fig. 3. Figure 3(a) presents the off-resonant Ta $L \alpha_{1}$ fluorescence spectrum, and Fig. 3(b) the reconstructed XAS spectra of the Ta $L_{3}$ edge. As shown, the effects related to the sample thickness are almost blanked by the HEROS approach. For all the investigated samples the observed relative deviations which do not exceed $4 \%$ are not related to the sample thickness but are due to the statistical fluctuations.

To compare the self-absorption effects around the Ta $L_{3}$ edge in the conventional fluorescence XAS and HEROS methods, the normalized fluorescence yields $(F Y)$ for two different thicknesses $d_{1}$ and $d_{2}$ of a target characterized by the total linear attenuation coefficient $\mu_{\text {tot }}$ were calculated using the following expression: 

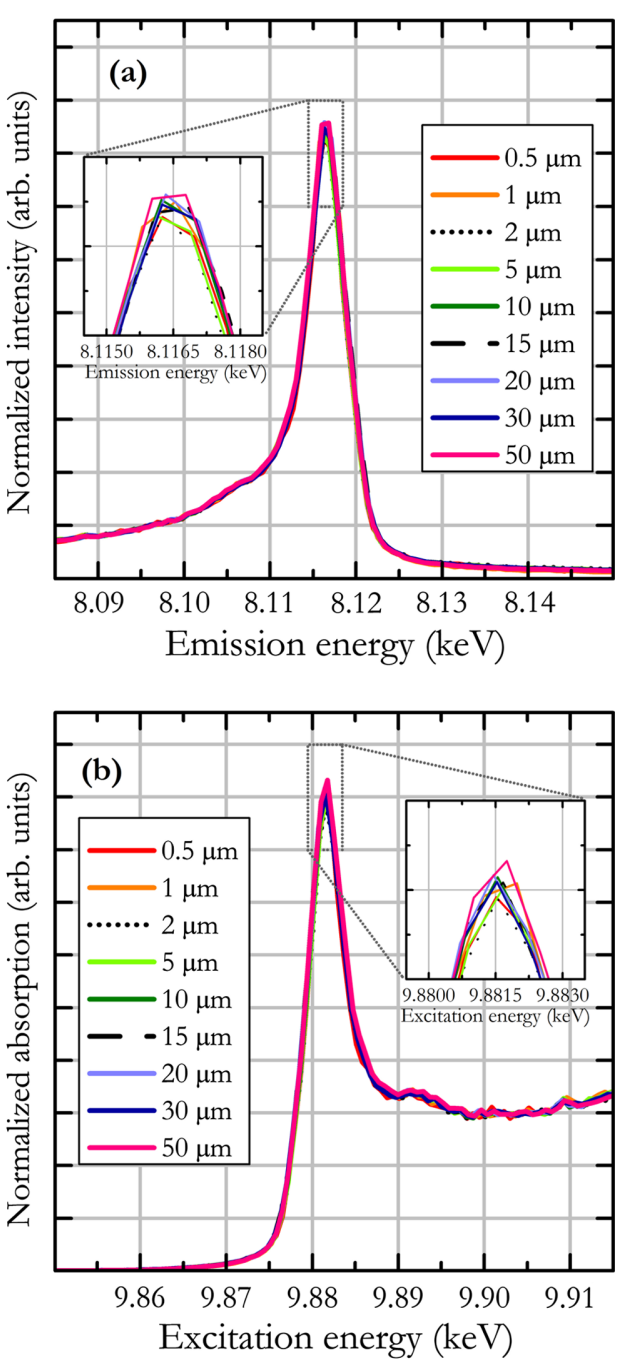

FIG. 3 (color online). Off-resonant XES spectra recorded for different sample thicknesses at an incident X-ray beam energy of $9.855 \mathrm{keV}$ (a) and the reconstructed HEROS spectra corresponding to the Ta $L_{3}$ absorption edge (b). The reconstruction was done by means of Eq. (1). The XES spectra were normalized to coincide at the emission energy of $8.096 \mathrm{keV}$ in (a) and the HEROS spectra at the excitation energy of $9.905 \mathrm{keV}$ in (b). The scatter of the data at the top of the two peaks for different sample thicknesses is shown enlarged in the insets.

$$
\frac{F Y\left(\hbar \omega_{1}, \hbar \omega_{2}, d_{1}\right)}{F Y\left(\hbar \omega_{1}, \hbar \omega_{2}, d_{2}\right)}=\frac{1-e^{-\left(\frac{\mu_{\mathrm{tot}}\left(\hbar \omega_{1}\right)}{\sin \phi}+\frac{\mu_{\mathrm{tot}}\left(\hbar \omega_{2}\right)}{\sin \theta}\right) d_{1}}}{1-e^{-\left(\frac{\mu_{\mathrm{tot}}\left(\hbar \omega_{1}\right)}{\sin \phi}+\frac{\mu_{\mathrm{tot}}\left(\hbar \omega_{2}\right)}{\sin \theta}\right) d_{2}}},
$$

where $\phi$ and $\theta$ are the incident and the exit angles with respect to the sample surface, respectively. The $\mu_{\text {tot }}$ was obtained by scaling the transmission mode XAS spectrum measured for the $0.5 \mu \mathrm{m}$ sample with the tabulated values of the absorption coefficient from Ref. [34]. Results of these calculations are compared in Fig. 4. In the conventional fluorescence mode XAS, the measured fluorescence yield depends on both the penetration depth (which depends on the incident beam energy and varies very
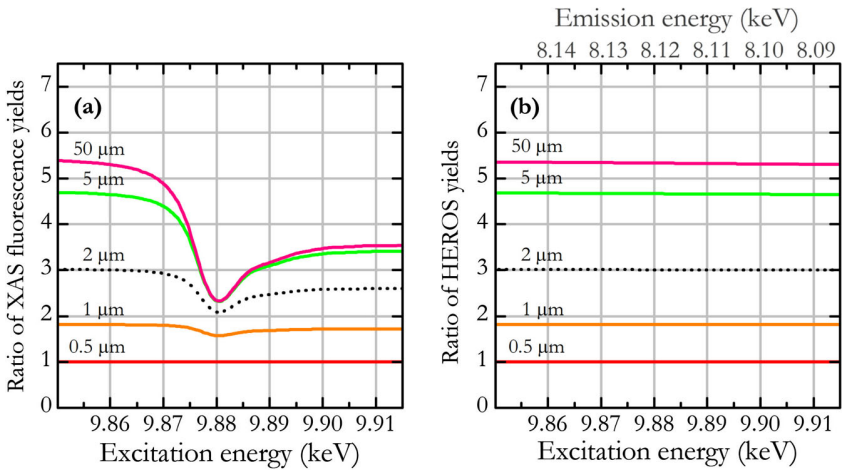

FIG. 4 (color online). Calculations showing the effects of the target thickness on the Ta $L_{3}$ edge in the conventional fluorescence mode XAS (a) and in the HEROS (b), for $\phi=\theta=45^{\circ}$. In the XAS case the calculations were performed for a fixed x-ray emission energy corresponding to the Ta $L \alpha_{1}$ line $(8.146 \mathrm{keV})$. In the HEROS case, the fluorescence yield ratios were first calculated. The calculations were performed for the beam energy used in the HEROS measurements $(26 \mathrm{eV}$ below the Ta $L_{3}$ edge). Then the energy scale of the x-ray emission was converted to the excitation energy scale using Eq. (1). For both XAS and HEROS, the yield ratios were calculated with respect to the $0.5 \mu \mathrm{m}$ thick sample (i.e., $d_{2}=0.5 \mu \mathrm{m}$ ).

strongly in the resonant regime) and the fluorescence escape length (which depends on the fluorescence radiation energy and varies slowly with the emission energy). As a result, thicker (or more concentrated) samples will provide stronger $F Y\left(\hbar \omega_{1}\right)$ signals, but will introduce stronger spectral distortions and reduction of the XANES peaks [see Fig. 4(a)]. It can be seen from the plots in Fig. 4 that the spectral intensity modulations in fluorescence mode XAS are not as much due to the self-absorption of the emitted fluorescence radiation but to the differences in the incident photon penetration depth as a function of the photon energy.

In contrast to the conventional fluorescence mode method, in the HEROS technique an XES spectrum is measured at a fixed off-resonant incident beam energy. The change of the target thickness, to a good approximation, introduces only scaling of the measured $F Y\left(\hbar \omega_{2}\right)$ [see Fig. 4(b)]. Processing the measured HEROS emission spectra by means of Eq. (1) yields absorption spectra whose shapes are independent of the target thickness [see Fig. 4(b)].

In the present work we employed the HEROS technique to measure the Ta $L_{3}$ edge absorption spectrum. The HEROS technique is based on retrieving the absorption spectrum from the off-resonant emission data collected at an incident beam energy detuned below the absorption edge under investigation. In contrast to conventional transmission and fluorescence mode spectra, the impact of self-absorption effects on the final XAS spectrum is reduced to only the probability of reabsorption of the produced fluorescence radiation in the target which is usually nearly constant in the measured emission energy 
range. This is confirmed by the reconstructed HEROS spectra which were found to be independent of the target thickness. We have demonstrated that for the Ta $L_{3}$ edge the HEROS method circumvents the problem of selfabsorption effects, potentially allowing the investigation of any strongly absorbing sample with fluorescence XAS. HEROS provides thus an alternative to existing XAS techniques, in particular when they are not applicable. It is the method of choice for single-shot XAS spectra measurements and for time-resolved high energy resolution XAS studies. It also represents a powerful tool to follow chemical reactions [22-24] and to extract precise information on the electronic structure from XAS spectra, which are free of self-absorption effects.

We wish to acknowledge the Swiss Light Source from the Paul Scherrer Institute for access to the SuperXAS beam line. Some of us (W. B., J. H., J.-Cl. D., and Y. K.) also acknowledge the financial support of the Swiss National Science Foundation.

*wojciech.blachucki@unifr.ch

${ }^{\dagger}$ Present address: Paul Scherrer Institute, CH-5232 VilligenPSI, Switzerland.

[1] A. Bianconi, J. Garcia, and M. Benfatto, Top. Curr. Chem. 145, 29 (1988).

[2] H. Wende, Rep. Prog. Phys. 67, 2105 (2004).

[3] J. Jaklevic, J. A. Kirby, M. P. Klein, A. S. Robertson, G. S. Brown, and P. Eisenberger, Solid State Commun. 23, 679 (1977).

[4] W. Gudat and C. Kunz, Phys. Rev. Lett. 29, 169 (1972).

[5] P. Eisenberger, P. M. Platzman, and H. Winnick, Phys. Rev. Lett. 36, 623 (1976).

[6] K. Hämäläinen, D. P. Siddons, J. B. Hastings, and L. E. Berman, Phys. Rev. Lett. 67, 2850 (1991).

[7] F. Reinhardt, B. Beckhoff, H. Eba, B. Kanngiesser, M. Kolbe, M. Mizusawa, M. Müller, B. Pollakowski, K. Sakurai, and G. Ulm, Anal. Chem. 81, 1770 (2009).

[8] A. Warland, C. Antoniak, M. Darbandi, C. Weis, J. Landers, W. Keune, and H. Wende, Phys. Rev. B 85, 235113 (2012).

[9] S. Landsberger, C. Brabec, B. Canion, J. Hashem, C. Lu, D. Millsap, and G. George, J. Environ. Radioact. 125, 23 (2013).

[10] M. Gräfe, E. Donner, R. N. Collins, and E. Lombi, Anal. Chim. Acta 822, 1 (2014) .

[11] R. Ortega, A. Carmona, I. Llorens, and P. L. Solari, J. Anal. At. Spectrom. 27, 2054 (2012).

[12] S. Bordiga, E. Groppo, G. Agostini, J. A. van Bokhoven, and C. Lamberti, Chem. Rev. 113, 1736 (2013).
[13] X. Chen, L. Liu, Z. Liu, M. A. Marcus, W.-C. Wang, N. A. Oyler, M. E. Grass, B. Mao, P.-A. Glans, P. Y. Yu, J. Guo, and S. S. Mao, Sci. Rep. 3, 1510 (2013).

[14] R. Mitzner et al., J. Phys. Chem. Lett. 4, 3641 (2013).

[15] S. Eisebitt, T. Böske, J. E. Rubensson, and W. Eberhardt, Phys. Rev. B 47, 14103 (1993).

[16] J. M. Ablett, J. C. Woicik, and C. C. Kao, in Advances in $X$-Ray Analysis : Proceedings of the Annual Conference On Application of X-ray Analysis, Denver, 2004, Vol. 48 (IEEE, New York, 2005), p. 266.

[17] C. H. Booth, F. Bridges, Phys. Scr. T 115, 202 (2005).

[18] A. J. Achkar, T. Z. Regier, H. Wadati, Y.-J. Kim, H. Zhang, and D. G. Hawthorn, Phys. Rev. B 83, 081106(R) (2011).

[19] A. J. Achkar, T. Z. Regier, E. J. Monkman, K. M. Shen, and D. G. Hawthorn, Sci. Rep. 1, 182 (2011).

[20] E. F. Aziz, M. H. Rittmann-Fran, K. M. Lange, S. Bonhommeau, and M. Chergui, Nat. Chem. 2, 853 (2010).

[21] F. M. F. de Groot, Nat. Chem. 4, 766 (2012).

[22] J. Szlachetko, M. Nachtegaal, J. Sa, J.-Cl. Dousse, J. Hoszowska, E. Kleymenov, M. Janousch, O. V. Safonova, C. König, and J. A. van Bokhoven, Chemical Comm. (Stockholm) 48, 10898 (2012).

[23] J. Szlachetko, J. Sá, M. Nachtegaal, U. Hartfelder, J.-Cl. Dousse, J. Hoszowska, D. L. A. Fernandes, H. Shi, and C. Stampfl, J. Phys. Chem. Lett. 5, 80 (2014).

[24] J. Szlachetko, D. Ferri, V. Marchionni, A. Kambolis, O. V. Safonova, C. J. Milne, O. Kröcher, M. Nachtegaal, and J. Sá, J. Am. Chem. Soc. 135, 19071 (2013).

[25] J. Tulkki and T. Åberg, J. Phys. B 15, L435 (1982).

[26] J. Szlachetko et al., Rev. Sci. Instrum. 83, 103105 (2012).

[27] A. Bergamaschi, A. Cervellino, R. Dinapoli, F. Gozzo, B. Henrich, I. Johnson, P. Kraft, A. Mozzanica, B. Schmitt, and X. T. Shi, J. Synchrotron Radiat. 17, 653 (2010).

[28] H. A. Kramers and W. Heisenberg, Z. Phys. 31, 681 (1925).

[29] Special issue on Progress in Resonant Inelastic X-Ray Scattering, edited by M. Simon and T. Schmitt [J. Electron Spectrosc. Relat. Phenom. 188, 1 (2013)].

[30] H. Hayashi, R. Takeda, Y. Udagawa, T. Nakamura, H. Miyagawa, H. Shoji, S. Nanao, and N. Kawamura, Phys. Rev. B 68, 045122(R) (2003).

[31] L. G. Parratt, C. F. Hempstead, and E. L. Jossem, Phys. Rev. 105, 1228 (1957).

[32] M. Bianchini and P. Glatzel, J. Synchrotron Radiat. 19, 911 (2012).

[33] E. A. Stern and K. Kim, Phys. Rev. B 23, 3781 (1981).

[34] J.H. Hubbell and S. M. Seltzer, http://physics.nist.gov/ xaamdi. 